\title{
Catalyst-Free Vapor-Phase Method for Direct Integration of Gas Sensing Nanostructures with Polymeric Transducing Platforms
}

\author{
Stella Vallejos, Isabel Gràcia, Eduardo Figueras, and Carles Cané \\ Instituto de Microelectrónica de Barcelona (IMB-CNM), CSIC, Campus UAB, Bellaterra, 08193 Barcelona, Spain \\ Correspondence should be addressed to Stella Vallejos; stella.vallejos@imb-cnm.csic.es
}

Received 25 April 2014; Revised 27 May 2014; Accepted 1 June 2014; Published 26 June 2014

Academic Editor: Shuangxi Xing

Copyright ( 2014 Stella Vallejos et al. This is an open access article distributed under the Creative Commons Attribution License, which permits unrestricted use, distribution, and reproduction in any medium, provided the original work is properly cited.

\begin{abstract}
Tungsten oxide nanoneedles (NNs) are grown and integrated directly with polymeric transducing platforms for gas sensors via aerosol-assisted chemical vapor deposition (AACVD) method. Material analysis shows the feasibility to grow highly crystalline nanomaterials in the form of NNs with aspect ratios between 80 and 200 and with high concentration of oxygen vacancies at the surface, whereas gas testing demonstrates moderate sensing responses to hydrogen at concentrations between $10 \mathrm{ppm}$ and $50 \mathrm{ppm}$, which are comparable with results for tungsten oxide NNs grown on silicon transducing platforms. This method is demonstrated to be an attractive route to fabricate next generation of gas sensors devices, provided with flexibility and functionality, with great potential in a cost effective production for large-scale applications.
\end{abstract}

\section{Introduction}

Advances in technology offer the opportunity to combine the properties of organic and inorganic materials to achieve electronic devices provided with flexibility and functionality. The availability of flexible sensing technologies, as chemoresistive gas sensors [1-3], with high functionality (i.e., sensitivity, selectivity, and stability) could offer a gateway to breakthroughs in distinct areas, for instance, in security, where detection of poisoning, explosive, or combustion gases is relevant and where wearable, light, cheap, and low-power consumption monitoring systems, yet not available in the market, are required. The availability of methodologies that allow the fabrication of sensors with flexible properties (e.g., fabricated in plastic, paper, or cloth) could have a great potential in a cost effective production for large-scale applications. Currently, combining flexibility and functionality implies a great challenge, as flexible materials (e.g., polymers) place stringent conditions on the production method of functional materials (e.g., nanoparticles (NPs) and nanostructured metal oxides (MOX)) requiring relatively low process temperature and limiting the use of some chemicals. Recent advances in organic chemistry have contributed to the production of high heat resistance polymers, such as polyimides, that withstand temperatures up to $400^{\circ} \mathrm{C}$ [4]. However and despite these advances, the integration of these polyimides with synthesis methods for producing highly crystalline materials, such as those based on vapor-phase $[5,6]$, still represents a technological issue to overcome, if nanotechnology is to be evolved in this line.

Some strategies towards the integration of nanostructured materials and flexible gas sensor devices have been described in the literature and could be separated in two main lines: indirect (post transfer) and direct methods. Post transfer methods are the most commonly reported and involve the transfer of nanostructured materials (previously prepared at high temperatures) on flexible substrates by dropcast (wet transfer) or by using stamps or soluble glues (dry transfer) $[1,3]$. Direct methods, in contrast, are infrequently reported and, generally, deal with hydrothermal processes due to the relatively low temperature required by this method $[7,8]$. Recently, a direct two-step method based on thermal oxidation was proposed $[9,10]$; however, the use of singlestep routes (e.g., vapor-solid approach without the use of catalyst seeding), despite potential fabrication advantages, is not reported. In this work we intend to move beyond the state of the art and address the issues present in the integration of synthesis methods for nanostructured materials and flexible 

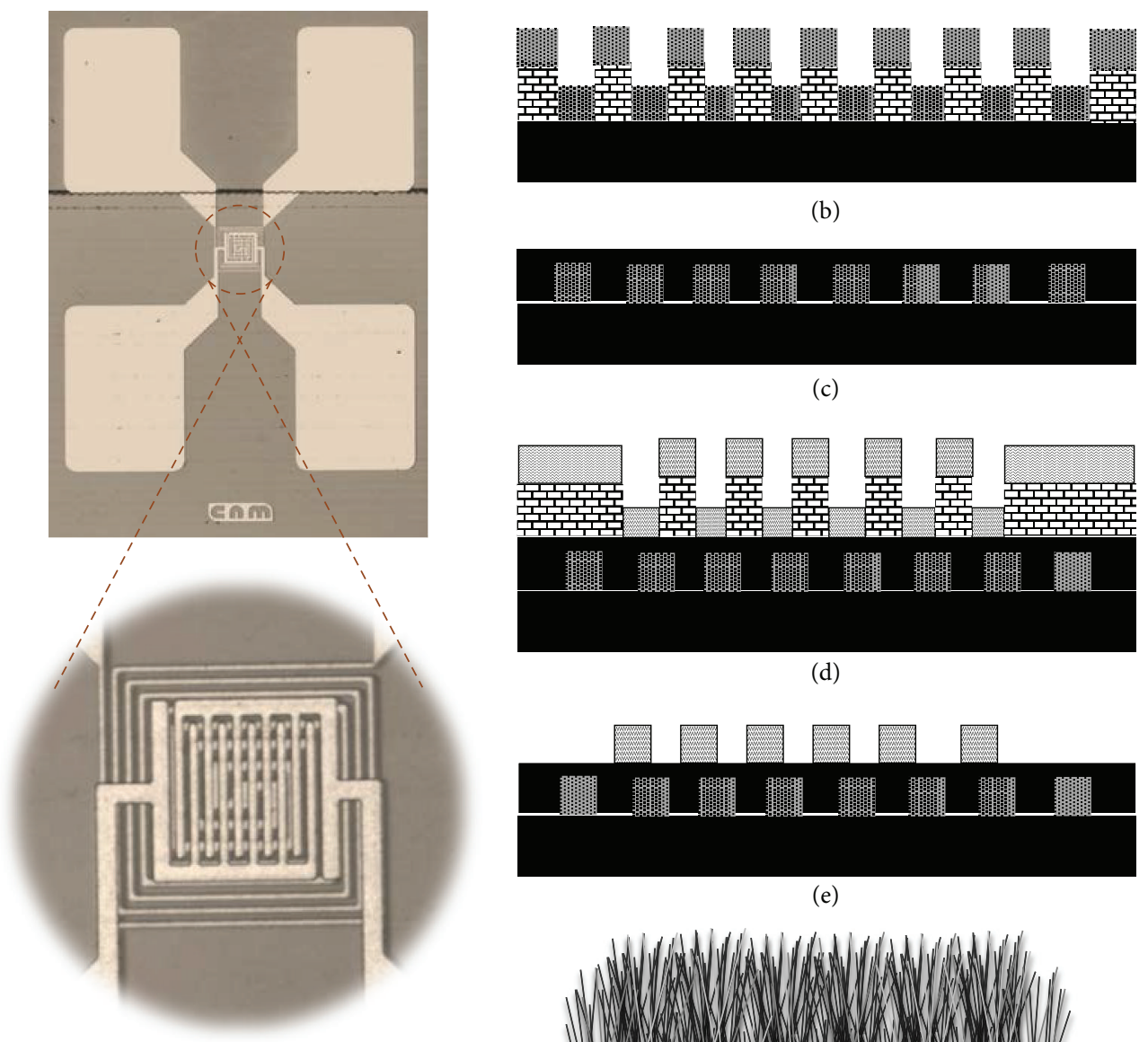

(b)

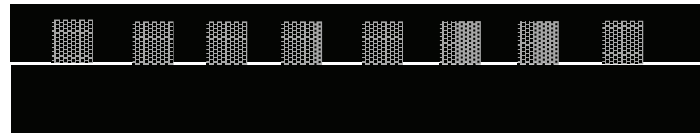

(c)

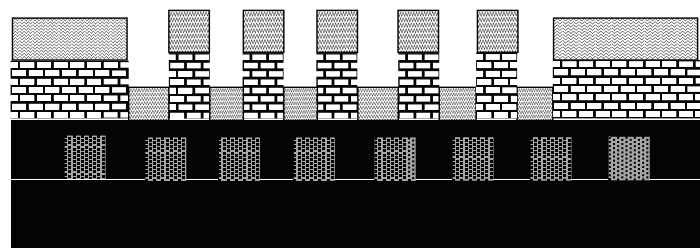

(d)

(a)

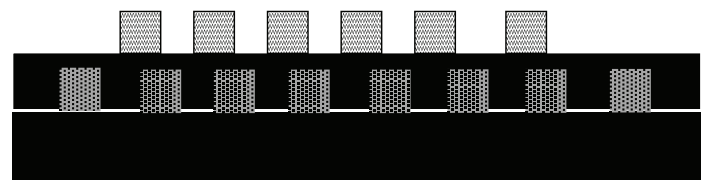

(e)

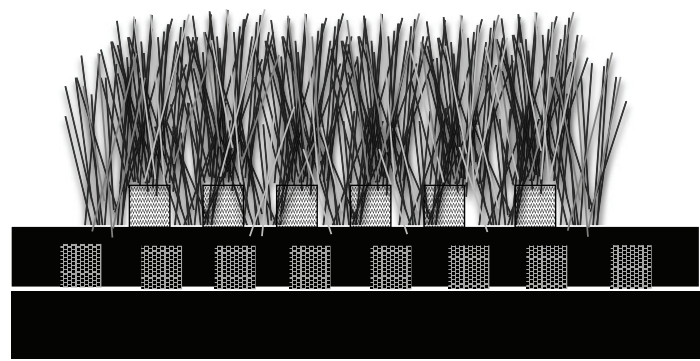

(f)

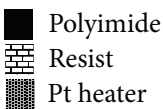

Pt electrodes
$\mathrm{NNs}$

FIGURE 1: Photograph of the polymeric transducing platform prior to growing the sensing nanostructures (a) and schematic view (not at scale) of the main technological processing steps involved in the fabrication of an individual gas sensor: photolithography, deposition, and lift-off of the Pt heater (b), deposition of an interlevel-polymeric layer (c), photolithography, deposition, and lift-off of the Pt electrodes ((d) and (e)), and catalyst-free direct growth of sensing nanostructures via AACVD (f).

devices using a method for synthesis of nanostructures in vapor-phase via AACVD, which has been demonstrated to be suitable for depositing, either nanostructures (e.g., $\mathrm{WO}_{3}$ ) [11] or thin films $\left(\mathrm{SnO}_{2}\right)$ [12] of $\mathrm{MOX}$ at relatively low temperatures (compared to other CVD methods for nanostructures synthesis).

AACVD is a variant of traditional CVD in which a precursor solution is transported to the substrate in an aerosol. The nucleation and growth kinetics of nanostructured materials and thin films are influenced by the deposition temperature and concentration of reactive species, which in turn influence the microstructure and thus the properties of the films. Nanostructured materials can be obtained by controlling the degree of homogenous and heterogeneous gas-phase reactions; hence by manipulating reaction conditions, deposition of nanostructured MOX can be achieved $[11,13,14]$. Synthesizing nanostructured materials in the gasphase has potential advantages over liquid phase synthesis including greater purity, continuous mode operation, and higher throughput but presents a number of challenges, including the controlled deposition of nanostructures onto surfaces and the chemical modification of individual nanostructures, to either passivate or functionalize their surface [15]. In this work, tungsten hexacarbonyl $\mathrm{W}(\mathrm{CO})_{6}$ was used for AACVD of tungsten oxide due to its lower decomposition temperature [16], compared to tungsten hexaphenoxide 

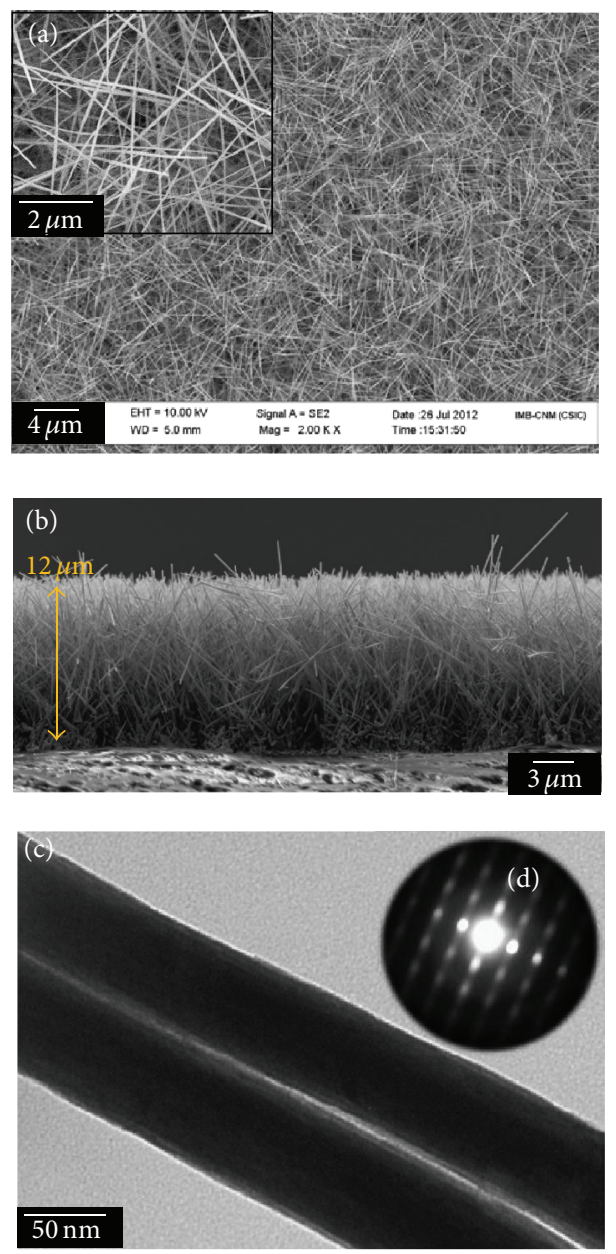

FIGURE 2: SEM and TEM imaging of tungsten oxide films with NNs-like morphology deposited on the polymeric transducing platform; (a) displays the film morphology from the top at low and high magnification, (b) displays the cross section of the films, (c) shows a pair of NNs removed from the polymer by sonication, and (d) shows the selected area electron diffraction of a NN.

$\mathrm{W}(\mathrm{OPh})_{6}[17]$ (the metallorganic precursor studied in our previous works) [11, 18]. Tungsten oxide is a material with promising properties for gas-sensor applications and has demonstrated high sensitivity to various gaseous species in particular $\mathrm{NO}_{x}[18,19]$, ethanol $[20], \mathrm{O}_{3}[19,20]$, and $\mathrm{H}_{2}$ $[18,21]$.

Here, we report for the first time the fabrication of flexible gas microsensors based on polymeric transducing platforms, their direct integration with tungsten oxide films in the form of NNs grown via AACVD, and their sensing properties towards hydrogen. These nanostructures have been integrated with the polymeric transducing platforms, without the use of intermediate steps such as wet or dry transfer of pregrown nanomaterials owing to the relatively low deposition temperatures required for synthesizing nanostructures in AACVD.

\section{Experimental}

Polymeric transducing platforms were fabricated using a commercial high heat resistant polyimide (Upilex-S, $125 \mu \mathrm{m}$,
UBE) to ensure thermal and chemical compatibility with AACVD process (Figure 1(a)). Clean room processes were carried out at wafer level and consisted of the following main technological steps: (1) photolithography, deposition, and lift-off of the platinum heater (Figure 1(b)), (2) deposition of an interlevel-polymeric layer (Figure 1(c)), and (3) photolithography, deposition, and lift-off of the platinum electrodes (Figures $1(\mathrm{~d})$ and $1(\mathrm{e})$ ). Photolithography processes were achieved using a reversible resist (AZ 5214E) to favor the subsequent lift-off processes. Platinum deposition was carried out using r.f. sputtering of a Pt target, whereas the interlevel-polymeric layer deposition and the opening of the heater contacts were achieved by spin coating of a thin layer of polyimide precursor (U-Varnish, UBE) and reactive ion etching, respectively.

Tungsten oxide films were grown on the top of polymeric transducing platforms via AACVD of tungsten hexacarbonyl at various temperatures $\left(300,325,350\right.$, and $\left.375^{\circ} \mathrm{C}\right)$. A commercial piezoelectric ultrasonic atomizer was used to generate an aerosol of the tungsten precursor $\left(40 \mathrm{mg}, \mathrm{W}(\mathrm{CO})_{6}\right.$, Sigma-Aldrich, $\geq 97 \%$ ) dissolved in methanol (10 mL, SigmaAldrich, $\geq 99.6 \%$ ). The aerosol droplets were transported to 
the heated substrate using a nitrogen gas flow $(200 \mathrm{sccm})$. The time taken to transport the entire volume of solution was typically about 70 minutes. A shadow mask was used during the deposition process, in order to protect the contacts and confine the film deposition to the electrode area (Figure 1(f)). After deposition, the structures were annealed at $375^{\circ} \mathrm{C}$ for $1 \mathrm{~h}$ in air. Additionally, the same AACVD procedure was used to deposit tungsten oxide films onto well-known silicon transducing platforms with the same transducer design [19], in order to have a reference sensor for gas testing.

The morphology and the elemental composition of the films were examined using Scanning Electron Microscopy and Energy Dispersive X-Ray Spectroscopy (SEM and EDX, Carl Zeiss, Auriga Series, $3 \mathrm{KV}$ ), the film structure was examined using X-Ray Diffraction (XRD, Bruker, AXS D8Advance, $\mathrm{Cu} \mathrm{K} \alpha$ radiation operated at $40 \mathrm{KV}$ and $40 \mathrm{~mA}$ ), and the chemical composition of the film was examined using X-Ray Photoelectron Spectroscopy (XPS, Phoibos 150 analyzer, SPECS GmbH, Berlin, Germany, in ultrahigh vacuum conditions (base pressure $1 \times 10^{-10} \mathrm{mbar}$ ) and a monochromatic $\mathrm{K} \alpha \mathrm{X}$-ray source, $1486.74 \mathrm{eV}$ ). Transmission Electron Microscopy and Selected Area Electron Diffraction (TEM and SAED, Jeol 1011, $100 \mathrm{kV}$ ) were performed by removing the film from the polymeric platform by sonication.

Gas sensors were tested in a continuous flow $(100 \mathrm{sccm})$ test chamber $\left(280 \mathrm{~cm}^{3}\right.$ volume). The system was provided with three mass flow controllers (Brooks 5850E), and mixtures of pure synthetic air and hydrogen $\left(\mathrm{H}_{2}\right.$, Praxair, $100 \mathrm{ppm}$ ) were adjusted in order to obtain the desired concentrations, as described previously [30]. Humidity was introduced to the system by bubbling through water under controlled conditions. To have a proper control of the relative humidity $(\mathrm{RH})$ inside the gas test chamber an evaluation kit (EK-H4, Sensirion AG) with a humidity sensor (SHT71, operating ranges from 0 to $100 \% \mathrm{RH}$, accuracy of $\pm 3 \% \mathrm{RH}$ ) was used. The sensors were exposed to hydrogen for different periods $(10,20$, and $90 \mathrm{~min})$ and subsequently the chamber was purged with air until initial baseline resistance was recovered. The whole testing period comprised $160 \mathrm{~h}$ during which sensors were tested to hydrogen concentrations at operating temperatures between 160 and $250^{\circ} \mathrm{C}$, performing up to 4 replicates for each condition. The sensor response ( $R$ ) was defined as $R=R_{a} / R_{\mathrm{H}_{2}}$, where $R_{a}$ is the sensor resistance in air at stationary state and $R_{\mathrm{H}_{2}}$ represents the sensor resistance after hydrogen exposure.

\section{Results and Discussion}

AACVD of $\mathrm{W}(\mathrm{CO})_{6}$ resulted in the formation of adherent uniform films confined on the electrode area of the polymeric and silicon transducing platforms. In general the films deposited at temperatures below $350^{\circ} \mathrm{C}$ were characterized by a dark black color, whereas films deposited at 350 and $375^{\circ} \mathrm{C}$ were characterized by a blue color. Previously, we have noticed that blue and dark black tungsten oxide films are indicative of partially reduced tungsten oxide $\left(\mathrm{WO}_{3-x}\right)$, with blue films characterized by formation of quasi-one-dimensional structures, such as NNs [11, 31].

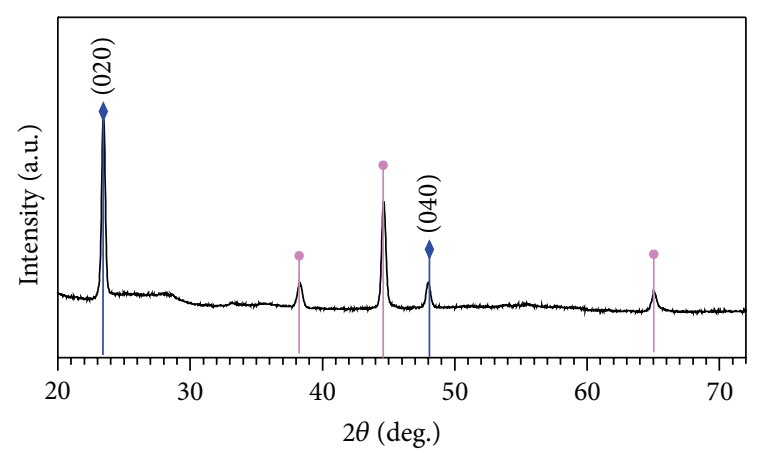

(a)

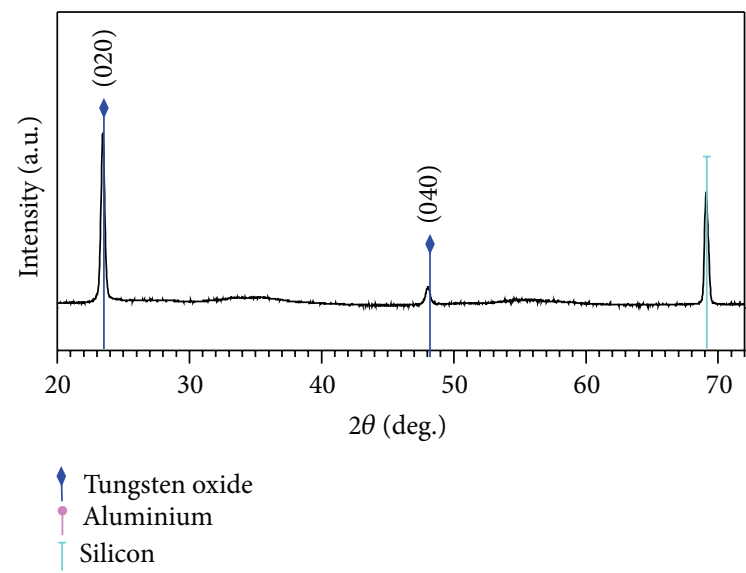

(b)

FIGURE 3: XRD patterns of the tungsten oxide NNs films deposited on polymer-based (a) and silicon-based (b) transducing platforms. Diffraction peaks at $23.5^{\circ}$ and $48.2^{\circ} 2 \theta$ in the data are indexed to a monoclinic phase (P21/n), ICCD card number 72-0677; the rest of the peaks are indexed to aluminium cubic phase coming from the sample holder and silicon.

Despite the relatively low sensitivity for oxygen determinations of EDX analysis, this technique indicated consistently $\mathrm{O} / \mathrm{W}$ ratios of $\sim 2.7$ in different points of the films, in both as-deposited and annealed films. Similarly, XPS analysis of the films deposited at $350^{\circ} \mathrm{C}$ indicated $\mathrm{O} / \mathrm{W}$ ratios of 2.8. The formation of tungsten oxide films from AACVD of W(CO) 6 is attributed to the use of oxygen-containing solvent, such as methanol, and the decomposition of $\mathrm{W}(\mathrm{CO})_{6}$ via the loss of the carbonyl group [31].

SEM imaging of the films, grown on polymeric and silicon transducing platforms (Figure 2(a)), showed a high density of nonaligned NNs with uniform diameters for films deposited above $350^{\circ} \mathrm{C}$, whereas films deposited below this temperature showed particle-like morphologies. As temperature exerts an important effect on the decomposition of the aerosol droplets, this parameter controls the resulting morphology via the type of chemical reactions during the deposition. Thus, if the temperature is low, heterogeneous reactions are dominant, resulting in the formation of dense films, whereas if the temperature is high, homogeneous reactions are dominant, resulting in the formation of fine particles. The formation of nanostructures such as NNs requires the coexistence of both 


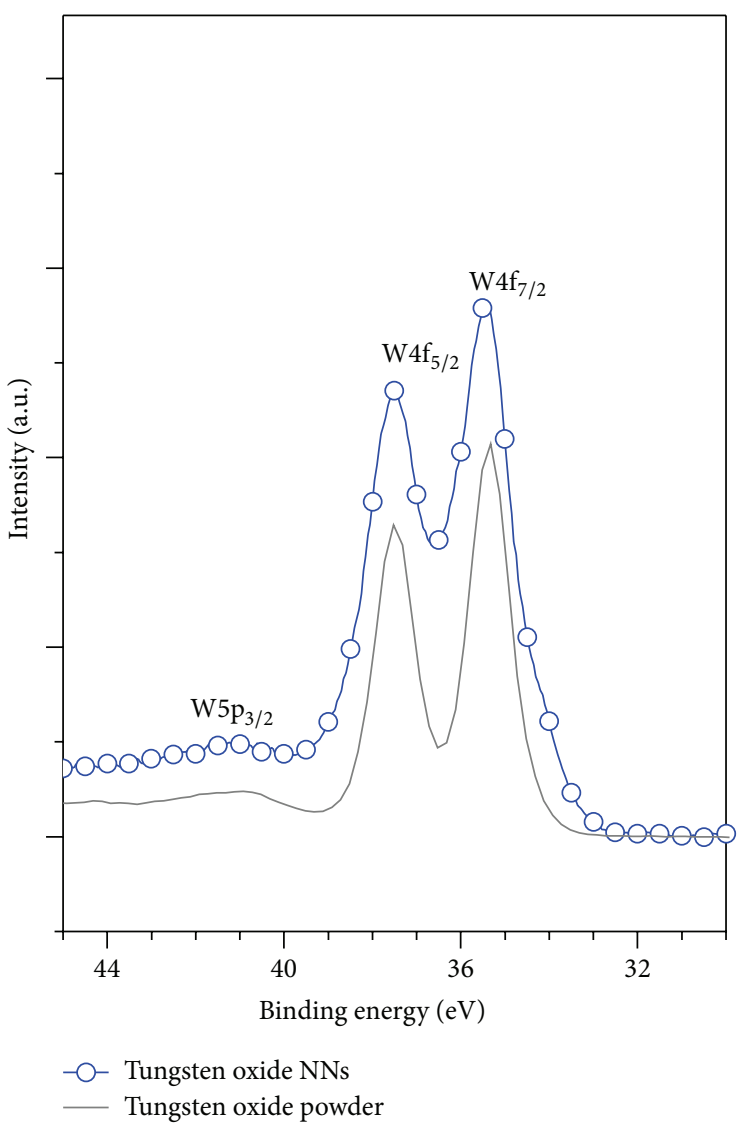

(a)

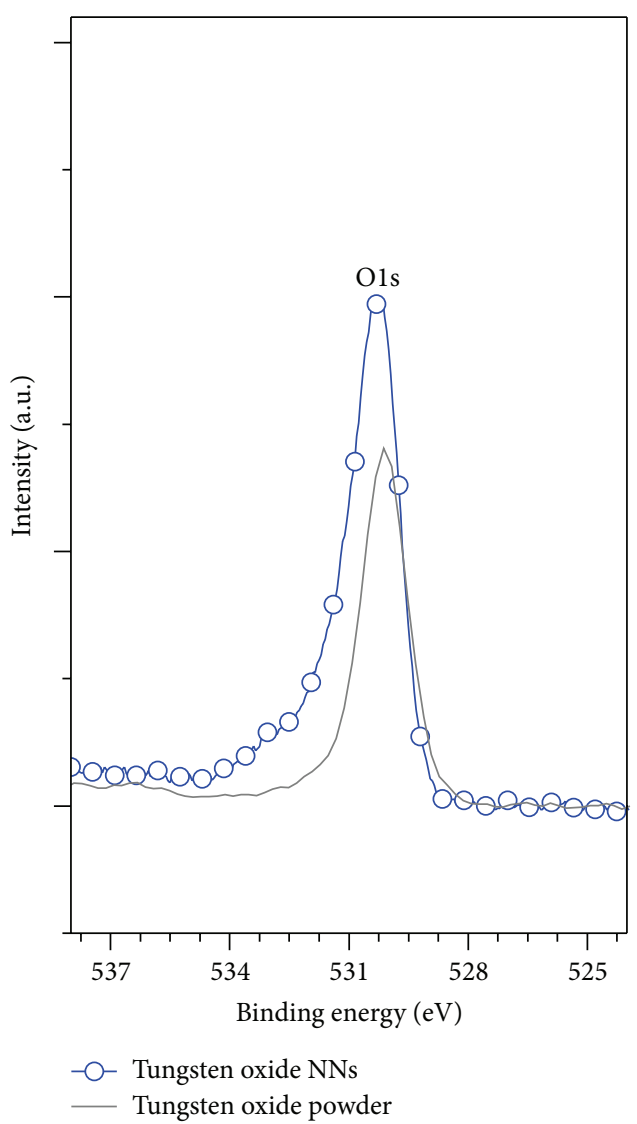

(b)

FIGURE 4: W4f (a) and O1s (b) XPS core levels spectrum recorded on tungsten oxide NNs grown onto the polymeric transducing platforms.

reactions in a similar degree $[11,15]$ and, in this work, this condition is achieved at relatively low temperature $\left(350^{\circ} \mathrm{C}\right)$ by using not only a precursor, that is, $\mathrm{W}(\mathrm{CO})_{6}$, with low temperature decomposition, but also a "carrier" solvent (i.e., methanol) which can act as a reactive precursor. Tungsten oxide NNs growth involves a catalyst-free direct vapor-solid mechanism; that is, the nanostructured films are formed from an initial polycrystalline layer grown at the initial step of the deposition, which provides not only energetically favorable planes for the nucleation of the NNs end caps, but also pathways for surface diffusion of subsequently condensed vapor species to the existing structure [5].

Cross section of the films grown on the polymer at $350^{\circ} \mathrm{C}$ (Figure 2(b)) showed randomly oriented NNs distributed uniformly along the section with lengths of $\sim 12 \mu \mathrm{m}$, and TEM (Figure 2(c)) of the same films showed the presence of NNs with diameters between 60 and $150 \mathrm{~nm}$, indicating the aspect ratio of the structures oscillates between 80 and 200. In addition SAED showed orientated diffraction patterns (Figure 2(d)) with spacing distance of $3.75 \pm 0.03 \AA$.

XRD analysis (Figure 3 ) of the films, comprised of NNs grown on polymeric and silicon transducing platforms, revealed the presence of monoclinic phase $\mathrm{WO}_{3}(\mathrm{P} 21 / \mathrm{n}$ space group, $a=7.306 \AA, b=7.540 \AA, c=7.692 \AA$, and $\beta=90.88^{\circ}$; ICCD card number 72-0677) and indicated a strong preferred orientation in the [010] direction, showing intense diffraction peaks at $23.5^{\circ} 2 \theta(d=3.77 \AA)$ and $48.2^{\circ} 2 \theta(d=1.88 \AA)$, corresponding to the (020) and (040) reflections of this monoclinic phase, which is consistent with similar results for AACVD of $\mathrm{W}(\mathrm{CO})_{6}$ on glass [31] and with the spacing distance observed by SAED. These results demonstrate the suitability of AACVD method for directly growing highly crystalline nanostructures on polymer-based devices.

Examination of the W4f and $\mathrm{W} 5 \mathrm{p}_{3 / 2}$ core level XPS spectrum of tungsten oxide NNs compared to a tungsten oxide powder standard (Figure 4(a)) shows similar peak positions, with a peak broadening in films comprised of tungsten oxide NNs likely associated with the presence of higher surface defects in these films $[32,33]$. The contribution of the surface defect becomes quantitatively important in the XPS spectrum due to the high energy excitation used and the higher surface area of these structures compared with the powder standard. Comparison of the O1s peaks of tungsten oxide NNs films and tungsten oxide powder standard (Figure 4(b)) suggests a slight shift between the peaks and the presence of a second component in the films comprised of NNs, which could be attributed to contamination on the samples [32].

Gas sensing tests of the polymer-based microsensors were carried out towards hydrogen, by using dc resistance measurements, at various sensor temperatures from 160 to 
TABLE 1: Summary of the gas sensing response and techniques employed to integrate sensing materials and flexible transducing platforms.

\begin{tabular}{|c|c|c|c|c|c|c|c|c|}
\hline Material & Method & Gas & $\mathrm{ppm}$ & $T_{\text {op }},{ }^{\circ} \mathrm{C}$ & $R_{g} / R_{a}$ & $t_{\text {res }}, \mathrm{s}$ & $t_{\text {rec }}, \mathrm{s}$ & Reference \\
\hline $\mathrm{WO}_{3}(\mathrm{NNs})$ & AACVD & $\mathrm{H}_{2}$ & 50 & 250 & 1.1 & 120 & 150 & This work \\
\hline $\mathrm{ZnO}$ (NWs) & Thermal oxidation & $\mathrm{H}_{2}$ & 500 & 280 & 11 & $<10$ & - & [9] \\
\hline $\mathrm{ZnO}$ (NRs) & Thermal oxidation & $\mathrm{NO}_{2}$ & 0.5 & - & 1.8 & - & - & {$[10]$} \\
\hline $\mathrm{ZnO}$ (NRs) & Hydrothermal & $\mathrm{EtOH}$ & 10 & 300 & 9 & - & - & {$[7]$} \\
\hline $\mathrm{Pd} / \mathrm{ZnO}$ (NRs) & Hydrothermal & $\mathrm{H}_{2}$ & 1000 & RT & 1.9 & - & - & {$[8]$} \\
\hline $\mathrm{ZnO}$ (NRs) & Thermolysis & $\mathrm{EtOH}$ & 100 & 300 & 3.11 & 237 & 318 & {$[22]$} \\
\hline $\mathrm{SnO}_{2}(\mathrm{TT})$ & Drop coating & $\mathrm{NO}_{x}$ & 2 & 325 & 23.8 & - & - & {$[23]$} \\
\hline $\mathrm{Pd} / \mathrm{SnO}_{2}(\mathrm{TT})$ & Drop coating & $\mathrm{EtOH}$ & 400 & - & 150 & - & - & {$[24]$} \\
\hline $\mathrm{WO}_{3}(\mathrm{TT})$ & Drop coating & $\mathrm{NO}_{2}$ & 5 & - & 200 & - & - & {$[24]$} \\
\hline $\mathrm{InO}_{x}$ & Sputtering & $\mathrm{O}_{3}$ & 0.02 & RT & 1.8 & - & - & {$[25]$} \\
\hline CNFs & Spray & $\mathrm{NH}_{3}$ & 500 & 220 & 1.3 & 300 & - & {$[26]$} \\
\hline CNT/graphene & Post transfer ${ }^{*}$ & $\mathrm{NO}_{2}$ & 5 & - & 1.1 & $>30$ & $>30$ & {$[27]$} \\
\hline CNT & Wet transfer & DMMP & 1 & RT & 4 & - & - & {$[28]$} \\
\hline $\mathrm{CNT}$ & Mech. abrasion & $\mathrm{NH}_{3}$ & 0.5 & - & $2-13$ & $>200$ & $>200$ & [29] \\
\hline
\end{tabular}

—: not specified, ${ }^{*}$ dry or wet post transfer (not specified), TT: thick film, NWs: nanowires, NNs: nanoneedles, NRs: nanorods, CNFs: carbon nanofibers, PECVD: plasma enhanced CVD, and RT: room temperature.

$250^{\circ} \mathrm{C}$ with intervals of $30^{\circ} \mathrm{C}$. Overall the baseline resistance of the sensors showed little variation $(\sim 1.3 \%)$ during the first stage of the testing period, approximately $120 \mathrm{~h}$ of working operation; after this period a change of the baseline resistance was noticed. SEM and XRD of the nanostructures after gas sensing test showed no alterations, suggesting the changes in the baseline resistance could be related with the aging of the microheater [34]. The thermal dependence of the sensor response towards hydrogen indicated a direct relation between the temperature and the response, although with a lower rate of change compared to our previous observations on tungsten oxide $[18,35]$. A comparison of the sensor response of tungsten oxide NNs, grown on the polymeric and silicon transducing platforms, revealed a comparable sensor behavior, with an approximately similar response $(\sim$ $120 \mathrm{~s})$ and recovery ( $150 \mathrm{~s})$ time (Figure 5) and a tendency to reach a stationary state, or a saturated sensor response, after $300 \mathrm{~s}$ of analyte exposure, with a complete recovery of the baseline resistance within $600 \mathrm{~s}$ after analyte removal. Results presented in Figure 6 show a direct dependence of the sensing response to relatively low hydrogen concentrations $(10,25$, and $50 \mathrm{ppm})$, with polymer- and silicon-based microsensors showing good reproducibility of the sensor response to each concentration (standard errors below $\sim 1 \%$ ). Similarly, humidity tests ( $\mathrm{RH} 14 \%$ ) to $50 \mathrm{ppm}$ of $\mathrm{H}_{2}$ showed good reproducibility with similar sensor response than those recorded for hydrogen in dry air.

A summary of the gas sensing results and the techniques employed in this work and the literature for the integration of sensing nanostructured materials and flexible transducing platforms is presented in Table 1. Although comparison of sensor outputs is complex, as sensor performance depends not only on the sensing material features but also on the fabrication and test parameters, we consider that these data are still meaningful to offer a general idea of the recent advances in this area. It is also worth noting that this table only refers to most common sensing materials (e.g., MOX,

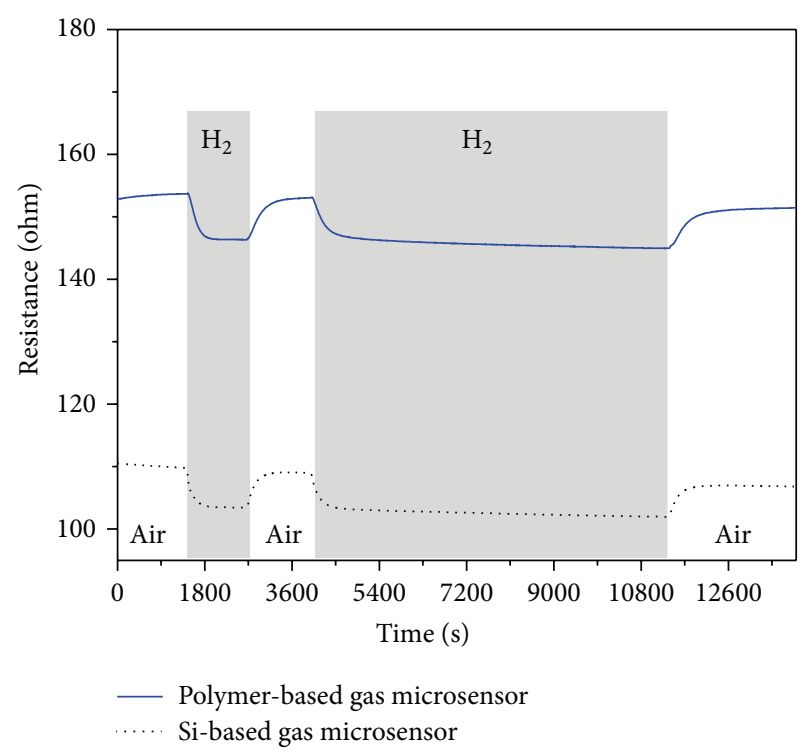

Figure 5: Film-resistance changes towards 20 and $90 \mathrm{~min}$ of hydrogen $(50 \mathrm{ppm})$ injection.

CNT) and not to those sensing films based on either polymers $[36,37]$ or hybrid structures [38, 39] (e.g., polymers mixed with carbon black). Most of the literature reporting the direct integration of MOX nanostructures on flexible transducing platforms has been based on hydrothermal processes [7, 8], and recently a direct two-step method based on thermal oxidation of a metal layer has been also proposed $[9,10]$. Overall, zinc oxide nanostructures have attracted much attention, particularly for direct integration approaches [7$10,22]$, whereas materials such as tin oxide, tungsten oxide, and indium oxide have been less used, with only few works reporting the use of these materials as thick or thin films integrated by wet transfer $\left(\mathrm{SnO}_{2}, \mathrm{WO}_{3}\right)[23,24]$ or sputtering 


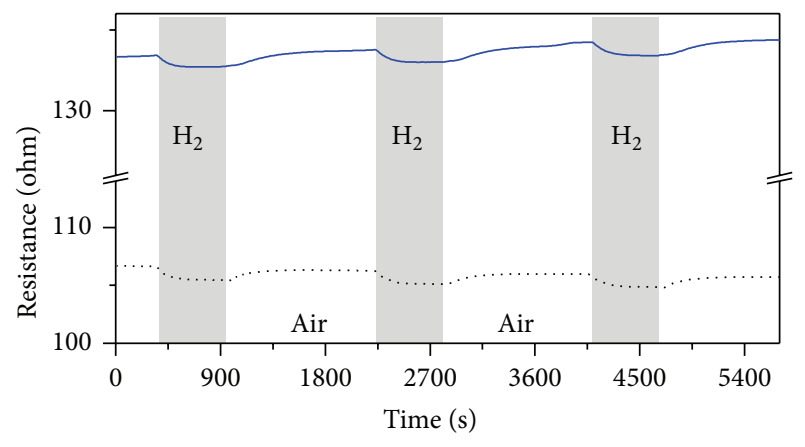

(a)

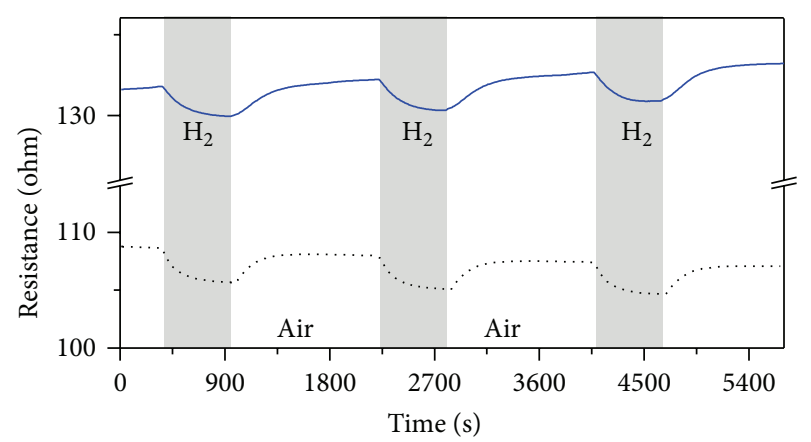

(b)

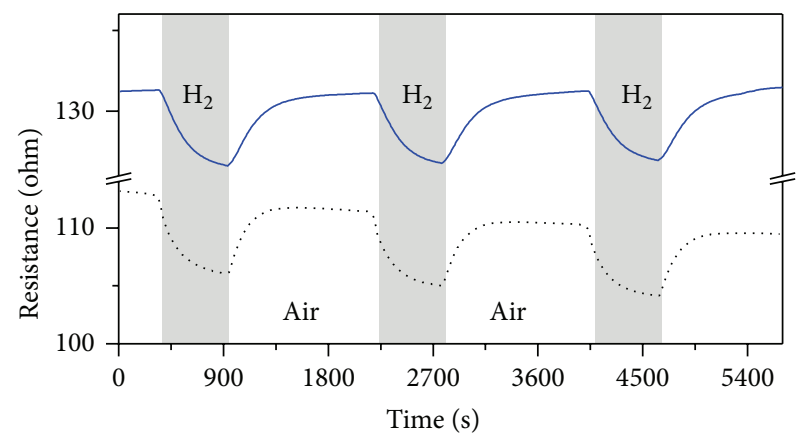

(c)

Figure 6: Film-resistance changes towards 10 (a), 25 (b), and 50 (c) ppm of hydrogen for the polymer-based (continuous line) and silicon-based (dot line) gas microsensors.

$\left(\mathrm{InO}_{x}\right)$ [25] method. Similarly, most of the works on carbon nanofibers [26] and carbon nanotubes [27-29] report the integration of these materials using post transfer methods. Despite potential advantages of gas-phase methods, such as AACVD, these have not been reported for the fabrication of flexible gas sensors yet. Table 1 also shows that the electrical resistance changes measured during the exposure of flexible gas sensors towards analytes have different orders of magnitude, with a tendency to relatively low sensor responses, except for flexible sensors based on thick films (rows 7-9). This might be related with the presence of residuals, coming from the different steps to transfer nanostructures onto the flexible substrate [27] or with the limitations to increase the sensor operating temperature in flexible devices [3].
These results show that the method presented in this work is a promising technique to integrate nanostructured MOX on polymer-based devices, with opposed characteristics to other integration methods such as hydrothermal or thermal oxidation, which require several processes steps, comparing to catalyst-free direct vapor-phase methods such as AACVD.

\section{Conclusions}

Gas sensors devices based on tungsten oxide NNs, synthesized and integrated directly with polymeric transducing platforms via AACVD, were fabricated and tested towards various concentrations of hydrogen. SEM and TEM results demonstrated the feasibility to grow highly crystalline nanomaterials in the form of NNs with aspect ratios between 80 and 200, whereas XPS suggested the presence of high concentration of oxygen vacancies at the surface of these structures. Gas testing demonstrated satisfactory sensing response of the polymer-based gas microsensors to hydrogen, at concentrations between $10 \mathrm{ppm}$ and $50 \mathrm{ppm}$, which are comparable to those obtained with tungsten oxide NNs grown on silicon transducing platforms. The method presented in this work is an attractive route to fabricate next generation of gas sensors devices, provided with flexibility and functionality, having advantages over other methods for integrating nanomaterials and devices, due to the relatively low processing temperature and no requirement for substrate pretreatment or post transfer steps.

\section{Conflict of Interests}

The authors declare that there is no conflict of interests regarding the publication of this paper.

\section{Acknowledgments}

The support of the Spanish Ministry for Science and Innovation via Grant TEC2010 21357 is gratefully acknowledged. Stella Vallejos Vargas also acknowledges the support of Juan de la Cierva Programme.

\section{References}

[1] M. C. Mcalpine, H. Ahmad, D. Wang, and J. R. Heath, "Highly ordered nanowire arrays on plastic substrates for ultrasensitive flexible chemical sensors," Nature Materials, vol. 6, no. 5, pp. 379-384, 2007.

[2] D. Briand, A. Oprea, J. Courbat, and N. Bârsan, "Making environmental sensors on plastic foil," Materials Today, vol. 14, no. 9, pp. 416-423, 2011.

[3] E. Comini, "Integration of metal oxide nanowires in flexible gas sensing devices," Sensors, vol. 13, no. 8, pp. 10659-10673, 2013.

[4] D.-J. Liaw, K.-L. Wang, Y.-C. Huang, K.-R. Lee, J.-Y. Lai, and C.-S. Ha, "Advanced polyimide materials: syntheses, physical properties and applications," Progress in Polymer Science, vol. 37, no. 7, pp. 907-974, 2012.

[5] Y. Liu and M. Liu, "Growth of aligned square-shaped $\mathrm{SnO}_{2}$ tube arrays," Advanced Functional Materials, vol. 15, no. 1, pp. 57-62, 2005. 
[6] J. Thangala, S. Vaddiraju, R. Bogale et al., "Large-scale, hotfilament-assisted synthesis of tungsten oxide and related transition metal oxide nanowires," Small, vol. 3, no. 5, pp. 890-896, 2007.

[7] J. Yi, J. M. Lee, and W. I. Park, "Vertically aligned $\mathrm{ZnO}$ nanorods and graphene hybrid architectures for high-sensitive flexible gas sensors," Sensors and Actuators, B: Chemical, vol. 155, no. 1, pp. 264-269, 2011.

[8] T.-R. Rashid, D.-T. Phan, and G.-S. Chung, "A flexible hydrogen sensor based on $\mathrm{Pd}$ nanoparticles decorated $\mathrm{ZnO}$ nanorods grown on polyimide tape," Sensors and Actuators, B: Chemical, vol. 185, pp. 777-784, 2013.

[9] M. Tonezzer and R. G. Lacerda, "Zinc oxide nanowires on carbon microfiber as flexible gas sensor," Physica E: LowDimensional Systems and Nanostructures, vol. 44, no. 6, pp. 1098-1102, 2012.

[10] D. Zappa, D. Briand, E. Comini, J. Courbat, N. F. de Rooij, and G. Sberveglieri, "Zinc oxide nanowires deposited on polymeric hotplates for low-power gas sensors," Procedia Engineering, vol. 47, pp. 1137-1140, 2012.

[11] S. Vallejos, P. Umek, and C. Blackman, "Aerosol Assisted Chemical Vapour Deposition control parameters for selective deposition of tungsten oxide nanostructures," Journal of Nanoscience and Nanotechnology, vol. 11, no. 9, pp. 8214-8220, 2011.

[12] T. T. Stoycheva, S. Vallejos, R. G. Pavelko, V. S. Popov, V. G. Sevastyanov, and X. Correig, "Aerosol-assisted CVD of $\mathrm{SnO}_{2}$ thin films for gas-sensor applications," Chemical Vapor Deposition, vol. 17, no. 7-9, pp. 247-252, 2011.

[13] K. L. Choy, "Chemical vapourdeposition of coatings," Progress in Materials Science, vol. 48, no. 2, pp. 57-170, 2003.

[14] W.-J. An, E. Thimsen, and P. Biswas, "Aerosol-chemical vapor deposition method for synthesis of nanostructured metal oxide thin films with controlled morphology," Journal of Physical Chemistry Letters, vol. 1, no. 1, pp. 249-253, 2010.

[15] S. L. Girshick, "Aerosol processing for nanomanufacturing," Journal of Nanoparticle Research, vol. 10, no. 6, pp. 935-945, 2008.

[16] T. T. Kodas and M. J. Hampden-Smith, The Chemistry of Metal CVD, Wiley-VCH, 1994.

[17] W. B. Cross, I. P. Parkin, S. A. O'Neill, P. A. Williams, M. F. Mahon, and K. C. Molloy, "Tungsten oxide coatings from the aerosol-assisted chemical vapor deposition of $\mathrm{W}(\mathrm{OAr})_{6}(\mathrm{Ar}=$ $\left.\mathrm{C}_{6} \mathrm{H}_{5}, \mathrm{C}_{6} \mathrm{H}_{4} \mathrm{~F}-4, \mathrm{C}_{6} \mathrm{H}_{3} \mathrm{~F}_{2}-3,4\right)$; photocatalytically active $\gamma$ - $\mathrm{WO}_{3}$ films," Chemistry of Materials, vol. 15, no. 14, pp. 2786-2796, 2003.

[18] T. Stoycheva, S. Vallejos, C. Blackman, S. J. A. Moniz, J. Calderer, and X. Correig, "Important considerations for effective gas sensors based on metal oxide nanoneedles films," Sensors and Actuators, B: Chemical, vol. 161, no. 1, pp. 406-413, 2012.

[19] S. Vallejos, V. Khatko, J. Calderer et al., "Micro-machined $\mathrm{WO}_{3}$-based sensors selective to oxidizing gases," Sensors and Actuators, B: Chemical, vol. 132, no. 1, pp. 209-215, 2008.

[20] A. Labidi, E. Gillet, R. Delamare, M. Maaref, and K. Aguir, "Ethanol and ozone sensing characteristics of $\mathrm{WO}_{3}$ based sensors activated by Au and Pd," Sensors and Actuators, B: Chemical, vol. 120, no. 1, pp. 338-345, 2006.

[21] R. Calavia, A. Mozalev, R. Vazquez et al., "Fabrication of $\mathrm{WO}_{3}$ nanodot-based microsensors highly sensitive to hydrogen," Sensors and Actuators, B: Chemical, vol. 149, no. 2, pp. 352-361, 2010.
[22] H. Ahn, J.-H. Park, S.-B. Kim, S. H. Jee, Y. S. Yoon, and D.-J. Kim, "Vertically aligned zno nanorod sensor on flexible substrate for ethanol gas monitoring," Electrochemical and Solid-State Letters, vol. 13, no. 11, pp. J125-J128, 2010.

[23] J. Courbat, D. Briand, L. Yue, S. Raible, and N. F. de Rooij, "Drop-coated metal-oxide gas sensor on polyimide foil with reduced power consumption for wireless applications," Sensors and Actuators, B: Chemical, vol. 161, no. 1, pp. 862-868, 2012.

[24] A. Oprea, J. Courbat, D. Briand, N. Bârsan, U. Weimar, and N. F. de Rooij, "Environmental monitoring with a multisensor platform on polyimide foil," Sensors and Actuators, B: Chemical, vol. 171-172, pp. 190-197, 2012.

[25] G. Kiriakidis, K. Moschovis, I. Kortidis, and R. Skarvelakis, "Highly sensitive InOx ozone sensing films on flexible substrates," Journal of Sensors, vol. 2009, Article ID 727893, 5 pages, 2009.

[26] S. Claramunt, O. Monereo, M. Boix et al., "Flexible gas sensor array with an embedded heater based on metal decorated carbon nanofibres," Sensors and Actuators, B: Chemical, vol. 187, pp. 401-406, 2013.

[27] H. Y. Jeong, D.-S. Lee, H. K. Choi et al., "Flexible roomtemperature $\mathrm{NO}_{2}$ gas sensors based on carbon nanotubes/ reduced graphene hybrid films," Applied Physics Letters, vol. 96, no. 21, Article ID 213105, 2010.

[28] Y. Wang, Z. Yang, Z. Hou et al., "Flexible gas sensors with assembled carbon nanotube thin films for DMMP vapor detection," Sensors and Actuators, B: Chemical, vol. 150, no. 2, pp. 708-714, 2010.

[29] K. A. Mirica, J. G. Weis, J. M. Schnorr, B. Esser, and T. M. Swager, "Mechanical drawing of gas sensors on paper," Angewandte Chemie-International Edition, vol. 51, no. 43, pp. 10740-10745, 2012.

[30] S. Vallejos, R. Cumeras, C. Calaza et al., "Localized heating to tungsten oxide nanostructures deposition on gas microsensor arrays via aerosol assisted CVD," in Proceedings of the 17th International Conference on Solid-State Sensors, Actuators and Microsystems (TRANSDUCERS \& EUROSENSORS XXVII), 2013 Transducers \& Eurosensors XXVII, pp. 1166-1169, 2013.

[31] S. Ashraf, C. S. Blackman, R. G. Palgrave, S. C. Naisbitt, and I. P. Parkin, "Aerosol assisted chemical vapour deposition of $\mathrm{WO}_{3}$ thin films from tungsten hexacarbonyl and their gas sensing properties," Journal of Materials Chemistry, vol. 17, no. 35, pp. 3708-3713, 2007.

[32] C. Navío, S. Vallejos, T. Stoycheva et al., "Gold clusters on $\mathrm{WO}_{3}$ nanoneedles grown via AACVD: XPS and TEM studies," Materials Chemistry and Physics, vol. 134, no. 2-3, pp. 809-813, 2012.

[33] S. Vallejos, T. Stoycheva, P. Umek et al., "Au nanoparticlefunctionalised $\mathrm{WO}_{3}$ nanoneedles and their application in high sensitivity gas sensor devices," Chemical Communications, vol. 47, no. 1, pp. 565-567, 2011.

[34] D. Briand, S. Colin, J. Courbat, S. Raible, J. Kappler, and N. F. de Rooij, "Integration of MOX gas sensors on polyimide hotplates," Sensors and Actuators, B: Chemical, vol. 130, no. 1, pp. 430-435, 2008.

[35] S. Vallejos, P. Umek, T. Stoycheva et al., "Single-step deposition of au- and pt-nanoparticle-functionalized tungsten oxide nanoneedles synthesized via aerosol-assisted CVD, and used for fabrication of selective gas microsensor arrays," Advanced Functional Materials, vol. 23, no. 10, pp. 1313-1322, 2013. 
[36] S. Ahlers, G. Müller, and T. Doll, "A rate equation approach to the gas sensitivity of thin film metal oxide materials," Sensors and Actuators, B: Chemical, vol. 107, no. 2, pp. 587-599, 2005.

[37] X. Liu, S. Cheng, H. Liu, S. Hu, D. Zhang, and H. Ning, "A survey on gas sensing technology," Sensors, vol. 12, no. 7, pp. 9635-9665, 2012.

[38] Y. Sun, S.-H. Hur, and J. A. Rogers, "Nano- and microstructured semiconductor materials for macroelectronics," in Springer Handbook of Nanotechnology, B. Bhushan, Ed., pp. 375-400, Springer, 2007.

[39] M.-T. Ke, M.-T. Lee, C.-Y. Lee, and L.-M. Fu, "A MEMS-based benzene gas sensor with a self-heating $\mathrm{WO}_{3}$ sensing layer," Sensors, vol. 9, pp. 2895-2906, 2009. 

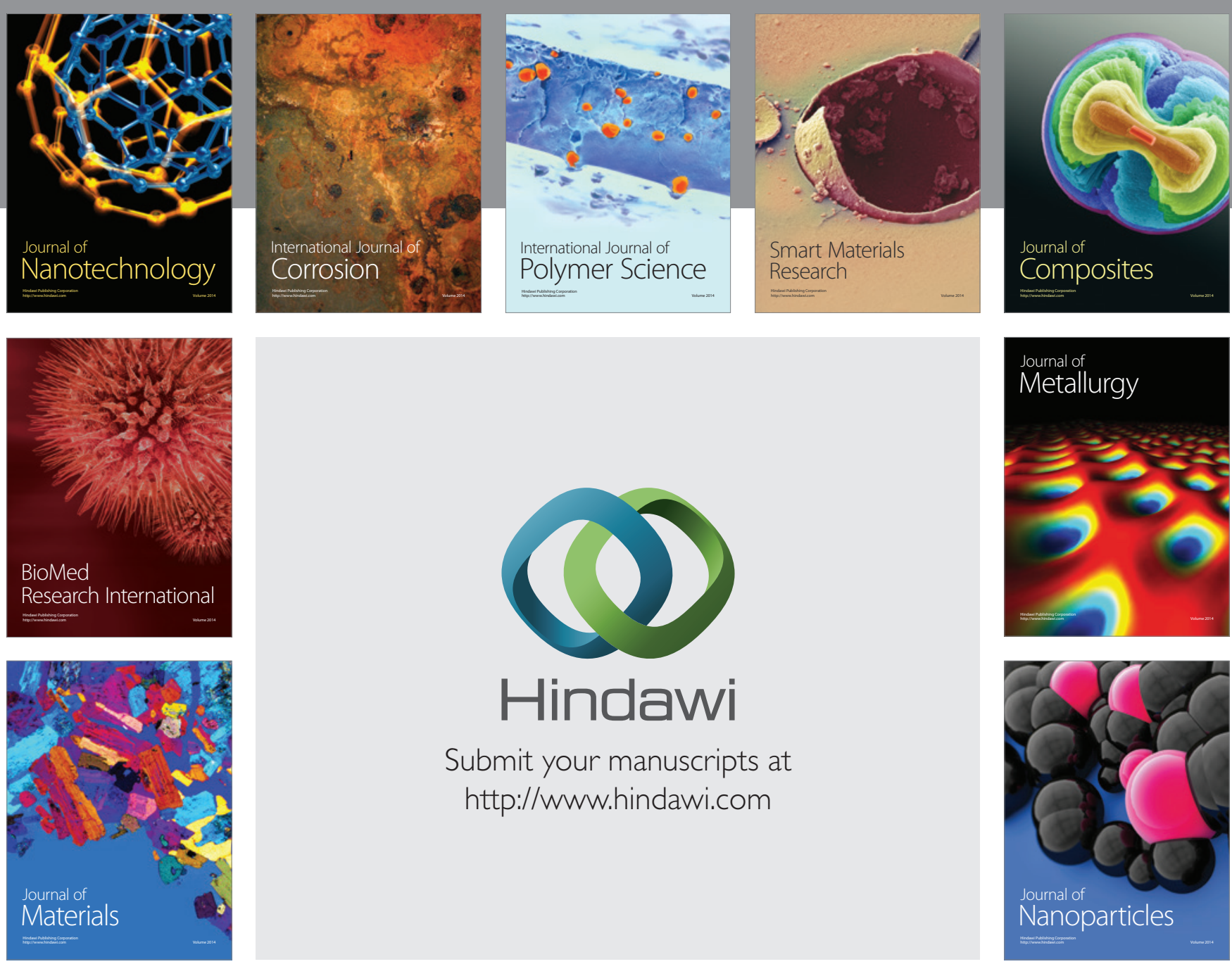

Submit your manuscripts at http://www.hindawi.com
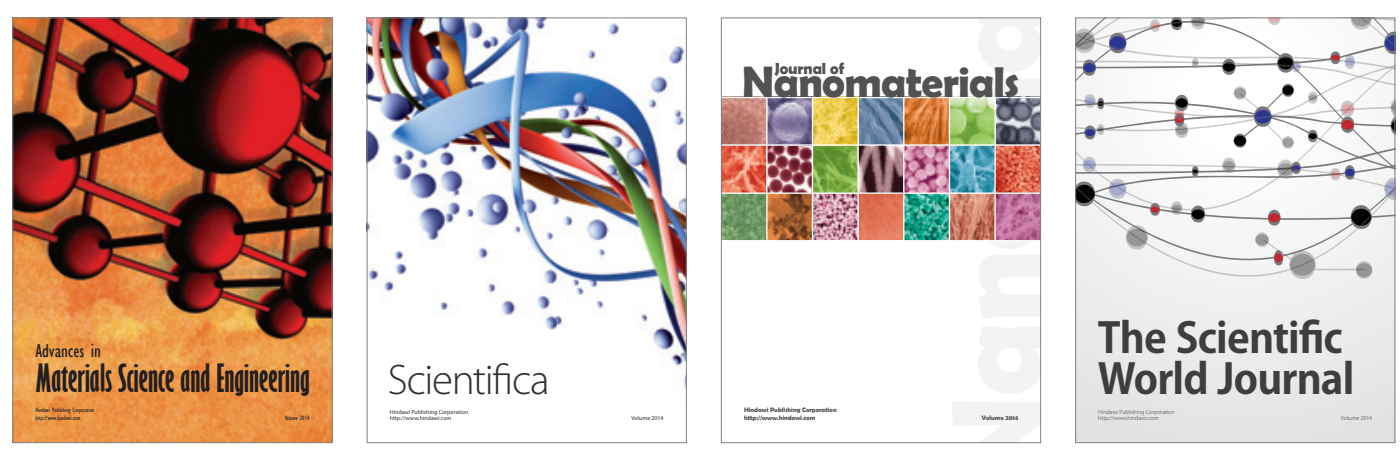

\section{The Scientific World Journal}
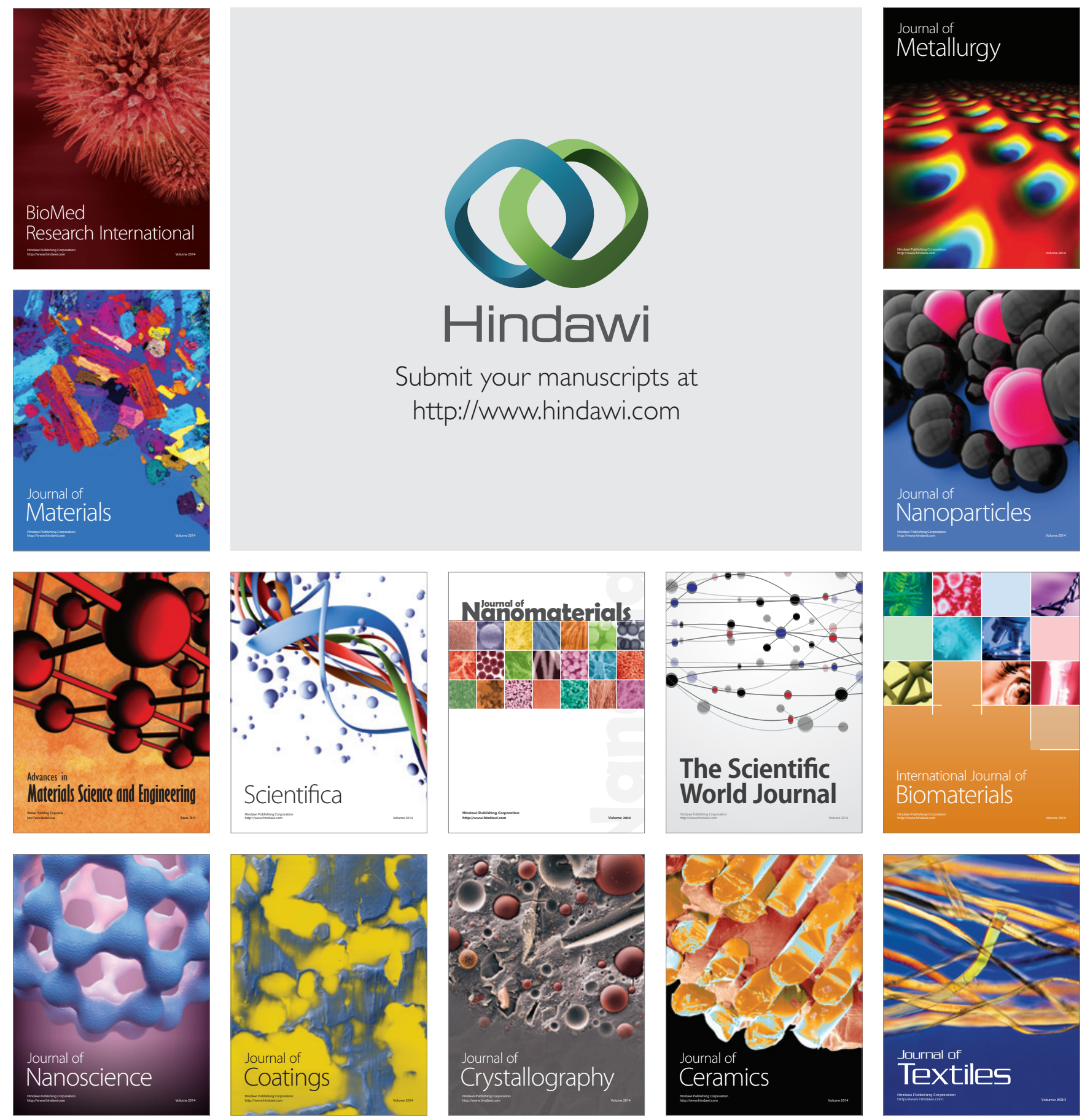